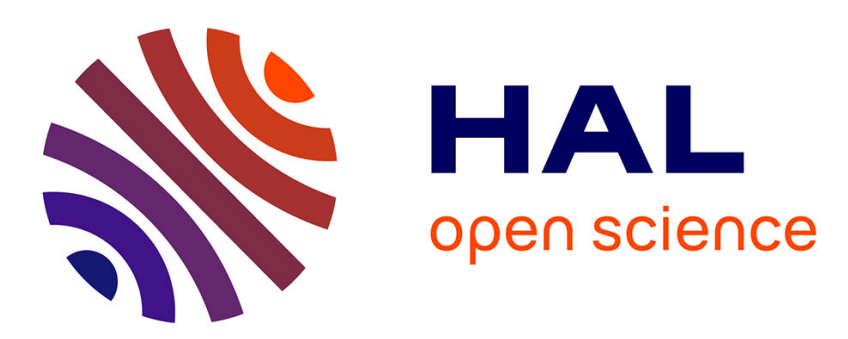

\title{
FAIRE VIVRE UN CLUB DE FOOTBALL EN ILE-DE-FRANCE : LE PARIS-SAINT-GERMAIN ET L'OLYMPIQUE NOISY-LE-SEC
}

Pascal Duret, Patrick Mignon

\section{- To cite this version:}

Pascal Duret, Patrick Mignon. FAIRE VIVRE UN CLUB DE FOOTBALL EN ILE-DE-FRANCE: LE PARIS-SAINT-GERMAIN ET L'OLYMPIQUE NOISY-LE-SEC. Les Annales de la Recherche Urbaine, 1998, 79, pp.119-125. hal-02045419

HAL Id: hal-02045419 https://hal-insep.archives-ouvertes.fr/hal-02045419

Submitted on 22 Feb 2019

HAL is a multi-disciplinary open access archive for the deposit and dissemination of scientific research documents, whether they are published or not. The documents may come from teaching and research institutions in France or abroad, or from public or private research centers.
L'archive ouverte pluridisciplinaire HAL, est destinée au dépôt et à la diffusion de documents scientifiques de niveau recherche, publiés ou non, émanant des établissements d'enseignement et de recherche français ou étrangers, des laboratoires publics ou privés. 


\title{
FAIRE VIVRE UN CLUB DE FOOTBALL EN ILE-DE-FRANCE : LE PARIS-SAINT-GERMAIN ET L'OLYMPIQUE NOISY-LE-SEC
}

\author{
Pascal Duret, Patrick Mignon*
} 119-125)

(Article paru dans: Annales de la recherche urbaine (0180-930X), 1998, $n^{\circ} 79$, "Sports en ville », pp.

Les compétitions sportives sont les lieux privilégiés de célébration des identités nationales ou d'identification à des collectifs locaux. Assister à un match de football permet de nouer des liens avec des semblables qu'on ne connaît pas (mais qui fréquentent le même stade) en discutant des mérites des jeunes joueurs, surtout quand ils sont des enfants du quartier ou de la ville. Les manifestations de fierté civique et d'appropriation de la ville liées à la vie sportive ne datent pas d'aujourd'hui; des défilés hebdomadaires d'ouvriers se rendaient au stade et aux messes du Labour célébrées tous les samedis après-midi que constituaient les rencontres de championnat, tandis que les finales de la Coupe d'Angleterre, présidées par le souverain, marquaient l'intégration dans la communauté nationale. Dans des villes comme Barcelone ou comme Bilbao, le club de football constitue un élément fondamental par lequel on prend conscience de son identité catalane ou basque, grâce auquel on apprend, à travers le jeu qui y est joué, les qualités morales du peuple auquel on appartient. Les analyses de Christian Bromberger sur Marseille, Naples et Turin montrent à l'œuvre le triangle qui lie un club, un stade et une communauté.

Or, signe des temps, les mots d'«insertion» et d'«intégration» deviennent de véritables les mots de passe des clubs, fussent-ils du plus haut niveau, pour trouver écho auprès des pouvoirs publics. L'existence, par exemple, de centres de formation dans les clubs professionnels français est présentée sous deux angles, un objectif sportif et un objectif social. Des clubs les plus élitaires aux clubs les plus populaires, tous se réclament de l'insertion, arguant que le stade contribue à donner à des groupes le sentiment d'une commune appartenance. Même lorsque les impératifs de communication s'imposent dans les politiques sportives locales, le football comme enjeu de culture participe de la mise en scène d'un corps social unifié, malgré des réalités contrastées comme celles du PSG et l'Olympique de Noisy-le-Sec, par exemple.

\section{La logique du PSG}

Contrairement aux autres grandes villes, Paris ne possède qu'un club professionnel de haut niveau, et encore ce club existe-t-il depuis un peu plus de vingt ans alors que les autres capitales sont représentées par des clubs qui sont nés au début du siècle. Les clubs professionnels parisiens n'ont pas résisté à la crise du football français des années 60 (crise financière des clubs et chute des spectateurs). De nombreuses hypothèses peuvent être proposées pour comprendre la situation.

On peut évoquer un professionnalisme tardif ou une urbanisation lente de la France qui n'oppose pas, jusqu'à une date récente, Paris à d'autres grandes villes (à l'exception de Marseille) mais plutôt un point de vue parisien à un monde provincial. On peut dire aussi qu'il n'y a pas de classe ouvrière parisienne capable de se mobiliser comme à Londres ou à Turin 
pour les équipes de football, ou alors qu'elle est trop diffuse, surtout regroupée dans des petites entreprises; que Paris est une ville interclassiste avec des quartiers ouvriers et des banlieues ouvrières mais toujours proches des quartiers bourgeois; que c'est une ville sous contrôle : elle n'a pas d'autonomie politique, donc pas de grandes familles, comme à Madrid ou à Milan qui se battent pour les suffrages des électeurs et financent des clubs de football, pas d'économie industrielle ou privée mais une économie administrative; que c'est une ville jacobine et politique : le sens de l'appartenance parisienne signifie qu'on se situe au-dessus des querelles de clocher, que la culture populaire vaut moins que l'engagement politique ou l'adhésion à la Culture, que la rivalité entre sport bourgeois et sport ouvrier disperse les amoureux du football dans les multiples stades de banlieues; que c'est une ville de l'ascension sociale et du détachement : on y va pour s'y perdre et pas pour retrouver un village; d'ailleurs les liens avec le village d'origine restent forts et si certains en ont la nostalgie, ils peuvent toujours soutenir les équipes qui les représentent plus que le club parisien; d'ailleurs, au moment où se construit le football en France ou lorsqu'il connaît son apogée, il y a peu de« vieux Parisiens ». Enfin, on pourra dire que l'air d'une capitale qui veut faire respirer l'universalisme fait qu'on aime à se penser individuellement en mouvement plutôt que collectivement installé sur des territoires. Il y a de toute façon toujours tension dans l'individu entre les différents pôles d'identification de l'origine culturelle, de la classe sociale, du quartier, etc. Ce qui nous donnerait une ville de l'attention distraite et cynique : on aime flâner sur les grands boulevards autant qu'aller au Parc ou au Vel' d'Hiv' ; on aime peut-être autant le spectacle que la victoire des siens et si c'est le cas, les siens ne sont pas nécessairement les Parisiens mais les Rémois dans les années 50-60 ou les Bretons et les Marseillais dans une période plus récente. Paris manifeste ainsi à l'extrême la relativisation du sport propre à la société française.

Ce qui manque au jeune Parisien pour trouver sa place dans le football ? D'abord une histoire (on compte près de vingt ans entre la descente du dernier grand club parisienne en 1965 et l'affirmation d'un nouveau), une tradition familiale (se déclarer pour une équipe), une routine (aller au stade toutes les semaines).

Une offre de football est faite dans les années 70. Le PSG de Daniel Hechter s'inscrit doucement dans le paysage du football français : il a au moins le mérite de perdurer. Mais il y a un tournant avec les années 80. L'élection du maire de Paris au suffrage universel à partir de 1979, et l'intérêt aussitôt manifesté par celui-ci pour le club de la capitale, puis l'arrivée d'une puissance comme Canal Plus, en 1991-92, font entrer Paris dans le régime commun à tous les clubs : être les premiers sur la scène sportive. Le public doit donc être mobilisé, comme source de revenus mais aussi comme douzième homme et comme partie intégrante de ce qu'est un grand club et une grande ville. La politique des stars (de Carlos Bianchi à Marco Simone), la filière des joueurs brésiliens, l'engagement, au début de l'existence du club, de joueurs s'adressant aux différentes catégories de spectateurs parisiens mobilisables, joueurs portugais, algériens, israéliens ou jeunes joueurs venant des clubs de banlieues en sont des manifestations comme les slogans adressés au public, "Ayez l'esprit de clocher !» ou «Venez célébrer la messe tous les samedis soirs au Parc des Princes», avant la saison 1991-92.

Les supporters doivent aussi au club l'ouverture d'espaces dans lequel inscrire leur action. En 1979, le président du club, Francis Borelli, lance une initiative pour mobiliser les jeunes spectateurs. Des places à prix très réduits sont proposées dans le virage Boulogne et des jeunes découvrent le Parc des Princes à cette occasion. Le kop de Boulogne naît ainsi à cette époque. Puis au début des années 90, le virage Auteuil. Au-delà des places bon marché, le club intervient pour la facilitation de la vie des supporters sous la forme de 
subventions, de locaux, d'aides aux déplacements. Le PSG se dote ainsi, en 1993-94, d'un département "supporter» puis d'équipes de stewards. II s'agit de gérer la question du hooliganisme et la mauvaise image du club diffusée par l'action de certains des membres du kop de Boulogne mais aussi de garder le contact avec ceux qui sont effectivement les plus fidèles des spectateurs ou favoriser l'apparition de nouvelles formes de supportérisme ultras dans de nouveaux espaces du stade, en l'occurrence le virage Auteuil. Cela traduit l'importance prise par les supporters, le fait que Paris est vraiment devenue une ville comme les autres, comme cela traduit le souci du club de contrôler son image.

II y a maintenant un public qui augmente au fur et à mesure de la montée en puissance du club car la fréquentation suit assez fidèlement les succès et les promesse de succès, la Coupe de France en 1981 et 1982 et le premier titre de champion en 1985-86, puis les aventures européennes : 17250 spectateurs de moyenne en 1975-76, 24000 en 1981-82 mais 16254 en 1984-85, puis 24571 en 1985-86; retombée à 14465 en 1990-91 pour remonter à 26600 en 1991-92 et un peu plus de 30000 pour la saison 96-97.

Qui a répondu à l'appel du club ? Si les données statistiques manquent sur les premières années, on peut au moins dessiner le profil du public du PSG de la première moitié des années 90 : c'est un public masculin à $90 \%$, venant de toute la région parisienne (20-30 $\%$ des spectateurs viennent de Paris) ; représentatif d'une région où les emplois tertiaires ont pris le pas sur les emplois industriels (34\% d'employés, $13 \%$ de professions intermédiaires et cadres moyens, $8 \%$ d'ouvriers) ; et jeune : $41 \%$ du public avait à l'époque moins de 24 ans et $64 \%$ moins de 35 ans. De visu, on peut également constater que le public est aussi fait de jeunes d'origine française comme d'immigrants portugais et espagnols, d'Africains, d'Antillais et de Juifs d'Afrique du Nord, mais aussi d'une partie du show-bizz ou du monde des médias qui aime se faire voir dans un endroit nouvellement à la mode. Malgré l'insuffisance des données, on peut dire qu'à l'exception des femmes et des Maghrébins, qui sont sous représentés par rapport à leur place dans la population de l'agglomération parisienne, le Parc des Princes est à l'image d'une région multiethnique où dominent les emplois du tertiaire.

Le public des virages, là où sont regroupés les supporters les plus engagés, n'est pas très différent. II est encore plus nettement masculin (moins de $8 \%$ de femmes) ; il est aussi un public de banlieusards (23\% seulement de Parisiens) ; il est enfin nettement plus jeune : $54 \%$ ont entre 15 et 24 ans, et $36.5 \%$ entre 25 et 34 ans; il est composé pour un tiers d'étudiants (34\%), tandis que près de la moitié exerce un emploi salarié (49\%). On note quelques différences toutefois entre les deux virages: Boulogne est un peu moins étudiant (30 contre $38 \%$ à Auteuil) ; il est aussi un peu plus vieux (39\% de 25-34 ans contre 24,3\% et $19 \%$ de 15-19 ans contre 24,3\%) à l'image de son antériorité. Ajoutons aussi que le public y est blanc, sous l'effet des engagements idéologiques de certains de ses occupants, ce qui n'est pas le cas d'Auteuil, d'une part parce que les ultras d' Auteui 1 attirent des supporters d'origine africaine ou maghrébine, d'autre part parce que ces ultras sont installés dans les tribunes populaires où on retrouve la mosaïque culturelle parisienne.

On peut référer la renaissance d'un public parisien à une nouvelle problématique de l'identité parisienne. La mobilisation autour du PSG et la composition du public des virages s'analysent comme des manifestations de la démocratisation de la jeunesse. Le football offre toute une gamme de possibilités identificatoires qui peuvent rendre compte du développement des groupes de supporters comme autant de formes d'action par lesquelles des groupes adolescents et post-adolescents mettent en scène leur rapport à la société, tentent de mettre en forme une expérience collective et gèrent l'attente et l'incertitude 
caractéristique de l'expérience juvénile, comme dans les souscultures rock ou rap. La création d'une équipe de football offre un champ à la recherche de différenciation, d'inclusion dans un ensemble plus vaste (une cause ou un mouvement) à ceux qui n'étaient justement pas impliqués dans les pratiques culturelles caractéristiques des jeunes des années 70 , que ce soit les concerts rock ou les boîtes.

L'engagement partisan pour une équipe renvoie aussi à une nouvelle problématique de l'identité parisienne. Certains groupes de supporters mobilisent explicitement la thématique parisienne quand ils s'appellent «Lutèce Falcon», les Gavroches ou «Titi-fosi», en plus des noms qui évoquent la localisation dans lc stade, "Boulogne Boys». La référence a un sens dans les règles du jeu des supporters, c'est une des conditions d'entrée : il s'agit bien de se donner des racines, de se définir contre les autres et d'arborer les couleurs du club qui sont celles de la ville, permettant ainsi l'identification immédiate en se posant face aux autres, tout en déclinant différentes thématiques.

Car dans les années de crise de son football, l'espace parisien s'est considérablement transformé. La France est vraiment devenue une société urbaine. Paris a perdu en partie son monopole de symbolisation de l'expérience urbaine revendiquée par d'autres métropoles, tandis que la dynamique sociale ou culturelle qui définit l'urbanité n'est plus seulement parisienne au sens strict, elle est devenue banlieusarde en même temps que les banlieues se sont aussi transformées.

Les transformations de l'espace parisien peuvent rendre compte d'une rupture avec la froideur du public parisien traditionnel et surtout d'un Paris devenu bourgeois. Etre fier d'être parisien, à travers l'occupation quasi hebdomadaire du quartier du Parc des Princes, est comme une protestation contre la forme bourgeoise de l'expérience parisienne que symbolise I'hostilité des riverains du Parc des Princes. II y a dans le supportérisme la manifestation qu'on n'appartient plus à ce monde parisien et qu'on revendique ce qui a été mis de côté : la passion, le populaire, le chaud, l'engagement contre la froideur, l'indifférence et la relativisation. L'équipe peut apparaître comme la représentation de ce qu'est le nouveau grand Paris, une ville ouverte où vivent des personnes de toutes origines. La banlieue y trouve alors une place. Les jeunes joueurs issus des clubs de banlieue ou du centre de formation du club sont des symboles de l'adéquation entre une équipe et ses supporters les plus fidèles qu'on présente composés de " petits ", étudiants, chômeurs ou détenteurs de petits boulots : des types des "quartiers» qui ont réussi. Les supporters se pensent comme des laissés-pour-compte de la société et du football. Le Parc des Princes joue à un double titre la promotion de la banlieue qu'on aimerait, pour certains, voir plus présente dans le football, dans l'équipe et dans le public.

\section{Le PSG vu des quartiers}

La manière dont le Parc des Princes est vu des banlieues alimente les représentations les plus caricaturales du PSG. II ne serait qu'un lieu propice aux provocations, où il convient d'arborer le drapeau algérien le plus près possible du kop de Boulogne, ou à la suite d'une victoire en Coupe d'Europe, de chercher l'affrontement avec les "fachos ". Le stade reconduirait l'expérience du divorce entre Paris et son centre. Faire le choix du PSG, ce serait faire le choix du centre, considéré par les jeunes des quartiers comme un monde hostile et paradoxalement " étranger ». Le football n'est pas nécessairement le club et l'équipe de la ville, c'est plus sûrement l'équipe de la cité et c'est aussi plus sûrement les Autres, le Brésil, les équipes africaines ou simplement les joueurs, Weah, Raï comme individus, mais surtout Marseille qui chez certains jeunes, du fait de la composition de l'équipe, de Tapie, des valeurs 
représentées par Marseille comme ville banlieue, etc., et du sens de l'opposition avec le centre de Paris.

Les supporters doivent au club des motifs de joie et de peine. II y a quelque chose d'excitant à participer au drame collectif du championnat ou de la Coupe d'Europe, d'attendre le résultat, d'apprécier la manière dont il a été obtenu, d'avoir enfin des émotions pour soi. De ce point de vue, la situation émotionnelle du supporter parisien est riche puisqu'il vit la tentative d'asseoir une réputation et de créer une continuité de jeu et d'esprit, et tous les aléas que cela comporte : des exploits mais aussi des résultats en dents de scie (un championnat presque sans défaite suivi par une menace de relégation) : des crises fréquentes dans le club entre joueurs et dirigeants : des erreurs administratives qui provoquent une défaite sur le tapis vert : des changements de style de jeu (offensif une saison, défensif l'autre) qui défient toute possibilité identificatoire (sommes-nous flamboyants ou sommes-nous efficaces ?) ; des vedettes qui ont fait le succès du club et qui sont vendues à d'autres : Les liens avec la région qui ne s'affichent pas suffisamment.

Si le PSG donne quelquefois la gloire, il donne aussi la honte et rend le supporter malheureux. Le supporter parisien est un supporter victime de ses dirigeants (ils ne font pas gagner à coup sûr), victime du décalage entre l'image que de nombreux supporters se donnent d'eux-mêmes et l'équipe (nous sommes pauvres, ils sont riches) mais victime aussi d'être parisien. Du coup, l'engagement et la fidélité du supporter n'en ont que plus de valeur car il doit surmonter les insuffisances du club. Mais en revanche, il peut se parer des vertus de ténacité, de travail et de droiture quand il peut accuser les clubs rivaux de devoir leurs succès aux « magouilles» comme à la belle époque de la rivalité avec le Marseille de Bernard Tapie

\section{La logique Noisy-le-Sec}

L'objectif majeur du club est double. D'une part, il recherche ouvertement l'accès le plus rapide possible au haut niveau (après une longue période de sommeil, il a franchi 10 catégories en 13 ans et se retrouve en Troisième division nationale). D'autre part, les dirigeants se fixent comme objectif l'insertion de tous leurs joueurs (pas uniquement ceux de l'équipe première).

En cela, le club remet en question les présupposés et les manières de faire sur lesquels reposaient les politiques municipales traditionnelles.

D'un point de vue idéologique, ses dirigeants ne croient pas au sport comme panacée miracle. Or traditionnellement le sport compétitif, parce qu'il se voulait intégration de la règle, était censé suffire pour assurer une socialisation de type "Troisième République» (Duret, Augustini, 1994), c'est-à-dire permettant l'intériorisation par les jeunes de normes achevées en provenance des adultes. Il était donc inutile de fixer des objectifs spécifiques d'insertion et d'intégration. S'y résoudre revient à reconnaître, d'une part, que le sport n'est pas socialisateur "par nature», et d'autre part, à se mettre en concurrence directe avec les services sociaux de la ville.

Du point de vue des mises en œuvre, les joueurs de l'équipe première de Noisy-le-Sec ne sont pas que des champions mais se doivent d'avoir aussi des compétences sociales. Les joueurs, qu'ils soient formés au club ou recrutés à l'extérieur, assurent la formation des plus jeunes, formation entendue au sens large de tutorat. Si depuis deux ans les joueurs de Noisyle-Sec sont rétribués (environ huit à dix mille francs par mois, ce qui est dérisoire en comparaison des salaires perçus au même niveau de pratique dans les autres équipes), leur contrat 
de travail mentionne explicitement ce volet social; ils doivent pour moitié leur gain à l'encadrement d'une équipe du club et pour moitié à leur qualité de joueur. Qu'il s'agisse de l'équipe réserve, vétéran ou d'une des sections de jeunes, le message se démarque nettement de celui du sport ouvrier (par exemple, de celui de la Fédération sportive gymnique du travail) : des plus jeunes au plus âgés, on vient rechercher la victoire. C'est en permettant à leur équipe de gagner sur le terrain que les entraîneurs espèrent résoudre la crise d'identité des habitants des cités, en leur redonnant fierté, en tirant parfois profit du sentiment qu'ils ont d'être des victimes et en faisant apparaître le match comme l'occasion d'une revanche.

La compétition sportive à Noisy-le-Sec consacre et exaspère une identité locale, aussi est-il essentiel que les jeunes puissent venir au club avec leur héritage culturel. La démarche est inverse à celle des grands clubs, comme le PSG, où on est prié par les dirigeants de laisser ses différences à l'entrée du vestiaire, ou des gradins(1), pour souder l'équipe et unir les spectateurs. A Noisy chacun est convié à faire profiter l'autre de son originalité culturelle : soirées couscous, soirée choucroute, soirée danse africaine se succèdent. Le programme des réjouissances est dérangeant pour le service des fêtes municipal, peu habitué à être dépossédé de l'organisation de la liesse populaire. Mais, parce qu'il n'est résolument pas communautaire, le club perturbe également les habitudes des associations de type Maccaby, AS berbère, AS portugaise où les sportifs se regroupent en fonction de leur appartenance religieuse ou ethnique.

Ces nouvelles formes d'engagement portées par la ferveur des jeunes de Noisy-le$\mathrm{Sec}$, non plus seulement envers l'équipe première mais envers l'ensemble du club, entraînent aussi d'importantes résistances municipales.

D'une part, le club par ses modes d'actions (en particulier les journée foot ouvertes à tous, y compris aux non licenciés), a mis en évidence des groupes qui n'étaient pas pris en compte par la ville. Ces centaines de jeunes font figure de laissés pour compte des politiques municipales. Ce constat entraîne des critiques réciproques entre les services municipaux et le club; la municipalité faisant valoir que les membres du club ne peuvent se contenter de mettre le doigt sur l'inadéquation entre l'offre de loisir et les demandes des jeunes sans servir également de force de propositions. Les municipaux essaient en outre de montrer les dirigeants comme se satisfaisant de leur position d'éternels demandeurs. Si on s'attache au registre argumentaire des plaintes formulées par les membres du club au sujet de leur difficulté de fonctionnement, on s'aperçoit effectivement qu'elles ont souvent un statut d'accusation des responsables municipaux. Mais ils reprochent avant tout à la mairie de leur faire un mauvais procès. lis font des propositions et plus encore ils les mettent en œuvre, mais dans des formes qu'elle n'est pas prête à accepter : accès au terrain des non-licenciés, pratiques artistiques et musicales éphémères associées aux événements sportifs, accès libre aux spectateurs. La dispute porte également sur les attributs qui symbolisent l'autorité et le pouvoir du « gardien» du gymnase : les clefs, le local à matériel. Autres sources de conflits, les refus de mise à disposition des bus municipaux pour transporter les équipes ou de mise en conformité réglementaire du stade par les services techniques avivent les tensions.

De plus, en essayant de maintenir coûte que coûte leur gestion familiale, les frères Sandjak résistent à la pénétration dans le comité directeur de représentants municipaux, toujours suspectés de vouloir faire du club «leur jouet». Au nom de l'intérêt des jeunes, l'esprit de famille est mis en avant. Face à l'animateur classique prompt à opposer des limites au crescendo de l'investissement professionnel pour conserver des plages réparatrices de vie privée, ces "entraîneurs-joueurs-animateurs-sociaux» s'en prennent aux "fonctionnaires" de l'action sociale. Ainsi, comme le fait remarquer l'un d'entre eux : « Dans les autres clubs ce 
qui leur plaît c'est d'avoir un grand club-house, avec le téléphone, la télé, et des bureaux pour recevoir. Moi je dis le bureau c'est vraiment pas important, les jeunes ils savent bien où j'habite et ça me dérange pas du tout, la cité c'est ma vie; et pour le club-house, l'essentiel c'est pas la couleur de la moquette, ce sont les horaires d'ouverture ". Derrière le côté matériel, ce qui est plus profondément mis en question ce sont les formes participatives des jeunes à la vie du club. A la gestion municipale jugée archaïque et centrée sur des intérêts extérieurs à ceux des jeunes, les Sandjak opposent un club géré par les jeunes et pour les jeunes. Ceux-ci font preuve d'un engagement précoce dans des tâches de responsabilité (comme l'accompagnement en déplacement ou l'organisation pendant les vacances scolaires de stages).

\section{Noisy-le-Sec face au PSG}

Comment Noisy-le-Sec peut-il faire face à ses adversaires avec des moyens financiers moindres ? La réponse consiste principalement à opposer la logique de la formation à la logique de l'achat. Le centre de formation est un enjeu crucial pour les petits clubs. Faut-il souligner que la nécessité de former les joueurs quand on ne peut pas les acheter se retrouve jusqu'en première division, quand on compare les budgets de fonctionnement des clubs de Lens ou de Metz par rapport à ceux du PSG de I'OM ou de Monaco. Mais à Noisyle-Sec la formation s'entend de surcroît au sens large; on ne souhaite pas façonner des " produits» compétitifs, mais permettre à des jeunes de se construire eux-mêmes à travers la vie du club.

Dans la logique compétitive classique incarnée par le PSG, le temps de l'entraînement est perçu en rupture avec le reste de la journée. Le joueur passe par le sas rituel des vestiaires où il est sommé par l'entraîneur de laisser ses problèmes (professionnels, familiaux. conjugaux...). et c'est en tant qu'avant-centre, arrière gauche, ou ailier qu'il entre sur le stade et cherche deux heures durant à s'améliorer. Ses liens avec les autres sections du club sont très réduits. Au mieux le joueur passe+il quelques minutes en exhibition devant les jeunes (comme le stipule son contrat) au pire il se contente de séances d'autographes. Son statut même de champion le coupe du reste du club.

Dans la seconde logique incarnée par Noisy-le-Sec, les dirigeants et les entraîneurs se refusent à ne voir dans le joueur qu'un rouage au service de la performance de l'équipe première. Dans ce mode de fonctionnement habituel dans d'autres disciplines (comme le rugby). mais à un niveau de jeu moindre, ce n'est plus uniquement le novice qui a le devoir de soutenir le champion, représentant des couleurs locales dans les compétitions de haut niveau, mais aussi le champion qui a celui d'assister le novice. Du coup, il existe une proximité bien plus importante entre les jeunes et les joueurs de l'équipe première. Le prestige du joueur de l'équipe fanion devenu manager ne manque pas de stimuler sur le terrain la fougue des jeunes. Mais son rôle s'étend à un tutorat qui concerne l'ensemble de la vie juvénile.

A la star inaccessible se substitue le " grand frère », dont la notoriété dans la cité est souvent préalable à la gloire sportive. II est important de constater, à partir du cas des frères Sandjak, que certaines familles dans les cités jouent un rôle décisif. II est donc sans doute vain de rechercher "le» grand frère idéal. Loin de réclamer l'unicité et la singularité, la fonction se partage ou se transmet de frères à frères. C'est la totalité de la famille qui bénéficie de l'ensemble du capital de prestige accumulé par chacun des frères, y compris de celui qui a fait le choix de partir jouer dans un club professionnel de première division, car il atteste en quelque sorte du niveau de jeu de ses autres frères. 
Même lorsqu'il poursuit des buts compétitifs, l'entraîneur de type "grand frère» se distingue de l'entraîneur de type haut niveau classique. D'une part, parce qu'il ne fait pas tendre le but final de tous les joueurs des équipes du club vers l'accès à l'équipe première (ou vers les services à rendre à l'équipe première). L'équipe 2, par exemple. ne se conçoit pas nécessairement comme une équipe " réserve» et peut poursuivre des buts qui lui sont propres. Ce n'est plus en se plaçant d'emblée dans un rapport de subordination avec les joueurs de l'équipe première que les jeunes donnent un sens à leur pratique personnelle. Pour autant, l'entraîneur " grand frère» veillera à l'esprit de famille du club par une diversité d'actions où le sport n'est qu'une séquence parmi d'autres (repas, rallyes...). D'autre part, il n'est pas seulement celui qui prépare l'équipe aux chocs dominicaux, il est encore celui qui partage les secrets et les problèmes de «ses» joueurs. Pourtant ses conceptions le distinguent radicalement de l'animateur social traditionnel. II n'est pas là pour faire du social; ce sont les jeunes qui viennent au club qui sont à la fois cibles du travail social et travailleurs sociaux.

Montrer son attachement au quartier comme à une grande famille constitue une condition essentielle à remplir dans cette logique. Péjorativement désignés comme «mercenaires», stigmatisation blessante à l'extrême dans le monde de l'honneur où les profits monétaires arrivent loin derrière les liens d'amitié, le joueur ou l'entraîneur ne peuvent quitter le club pour un meilleur contrat, sans passer pour "traîtres» aux leurs. Inversement entraîner ou jouer pour un club dans la logique du haut niveau spectaculaire suppose d'être " sans attache », condition essentielle pour être considéré comme un "vrai pro» et comme un " mercenaire» formule qui passe alors pour un compliment recherché.

Remplacer la star inaccessible par le " grand frère » contribue à relativiser les exploits de la vedette " en image » au profit du champion «en chair et en os». La télévision, et plus encore les vidéo-cassettes opèrent des montages où ne sont retenus que des exploits brévissimes mais répétés, offrant l'impression trompeuse que les stars sont infaillibles et infatigables. Jamais Bebetto. Raï ou Ronaldo ne semblent souffrir. L'image, en gommant les efforts, accentue le côté ludique, le don et le génie et corrélativement masque les souffrances et les efforts qui sont la condition pour accéder au plus haut niveau. La présence en chair et en os d'un champion permet d'estimer àsa plus juste valeur les progrès dans la pratique.

\section{Les différences dans les relations villes-club}

Alors que les formes d'engagement derrière les couleurs du PSG sont inégales, la mairie de Paris intègre pourtant l'existence de l'équipe dans sa politique de communication; à l'inverse, Noisy-le-Sec, qui suscite une adhésion sans faille des spectateurs locaux, n'est pas suivi par sa municipalité. Pour expliquer cette énigme, il convient de rappeler que le PSG ne se sert de la ville et ne la sert que pour son image. Le club de Noisy-le-Sec, plus modeste d'un point de vue compétitif, a pourtant des objectifs sociaux plus ambitieux qui supposent que la mairie, pour le suivre, s'aventure en terrain inconnu. Une nécessaire mise en confiance réciproque serait sans doute à construire entre les frères Sand jack et les responsables municipaux.

Le rapprochement entre municipalité et club dans les banlieues n'est pensable qu'au prix de la reconnaissance de la multiplicité des références et des pouvoirs pertinents. Entraîneurs et cadres municipaux doivent accepter de partager leurs compétences et admettre qu'ils ont mutuellement besoin de leurs savoir-faire, s'organiser pour vouloir faire ensemble. 
Les deux logiques, celles du PSG et celle de Noisy-leSec, activent chacune à leur manière les rapports sociaux fondamentaux. Pour vivre en société, il faut se savoir soutenu, savoir qu'on peut compter sur les autres et dans quelle mesure. Mais il faut aussi se sentir contenu, c'est à -dire avoir le sentiment des limites et de l'étendue de ses possibilités dans l'interdépendance d'un collectif. Dans tous les cas, l'investissement des autres sur soi conditionne son propre investissement envers les autres. Dans les grands clubs les relations sociales passent par le spectacle et l'animation des supporters. L'identification à l'équipe la transforme en surface de projection des qualités emblématiques de la ville. Les supporters reconnaissent aux joueurs des qualités qu'ils s'attribuent à eux mêmes. Donner une valeur à son existence est laissé à la charge de l'équipe que l'on soutient. Les jeunes y gagnent une tradition et une histoire. mais en retour se retrouvent dépendants. Dans un espace comme celui de Noisy-le-Sec, le lien social ne peut être pris en charge par la seule identification à l'équipe première. Les jeunes ne se sentent exister dans le regard des autres qu'en tant qu'acteurs.

Ces deux logiques correspondent à des situations urbaines très différentes, qui n' ont pas à être opposées l'une à l'autre seulement à cause de la différence de résultats sportifs qu'elles connaissent actuellement. L'une comme l'autre concourent à l'intégration des jeunes, sur des modes accordés à leurs cas respectifs. Si le spectacle télévisuel tend à conformer la perception du match de football à une norme centrale. la diversité des situations locales donne des réalisations concrètes du sport une image plurielle.

\section{NOTES}

1. Cependant, on doit à $\mathrm{C}$. Bromberger d'avoir parfaitement montré que malgré les discours consensuels des dirigeants, les supporters soutenant une même équipe ne constituent nullement une collectivité harmonieuse, mais se livrent entre eux à une âpre concurrence. 